\title{
A mathematical model of ternary fuzzy set for voting
}

\author{
Chao Wang ${ }^{\mathrm{a}, *}$, Minghu $\mathrm{Ha}^{\mathrm{a}}$ and Xiaowei Liu ${ }^{\mathrm{b}}$ \\ ${ }^{a}$ School of Economics and Management, Hebei University of Engineering, Handan, P.R. China \\ ${ }^{\mathrm{b}}$ School of Science, Hebei University of Engineering, Handan, P.R. China
}

\begin{abstract}
Intuitionistic fuzzy sets can effectively deal with the support information, opposition information and abstention information of candidates in many voting. However, it may be hardly to handle some information of voting for non-candidates in some voting. In order to handle the aforementioned problem, the concept of ternary fuzzy set and ternary fuzzy number are defined, and the corresponding operations are given and discussed. Then, a mathematical model for voting is established based on the ternary fuzzy set and ternary fuzzy number, the effectiveness of the model is verified by an example.
\end{abstract}

Keywords: Voting, intuitionistic fuzzy set, ternary fuzzy set, ternary fuzzy number

\section{Introduction}

Fuzzy set introduced by Zadeh [9] in 1965 is an important extension of classic set, and has been applied into many areas of modern life $[6,10,13]$. The membership degree of fuzzy sets can to describe the support information of voting $[11,16]$. However, not only support information but also opposition information and abstention information exist in voting. In 1983, Atanassov [7, 8] extended the fuzzy set and proposed the concept of intuitionistic fuzzy set. Each element of intuitionistic fuzzy set is described by the membership degree and nonmembership degree, and then the indeterminacy degree was given, which can describe the abstention information. Due to the advent of the theory, more and more scholars engaged in the research of intuitionistic fuzzy set. Atanassov [8] and De [12] defined and discussed the basic operations of intuitionistic fuzzy set. Xu [17, 18] further developed the intuitionistic fuzzy set theory, systematically studied

\footnotetext{
${ }^{*}$ Corresponding author. Chao Wang, School of Economics and Management, Hebei University of Engineering, Handan 056038, P.R. China. Tel.: +86 1510 3102799; Fax: +86 0310 8579089; E-mail: wang222chao@163.com.
}

the information aggregation approach of intuitionistic fuzzy set, and applied into multi-person multi-attribute decision making models under intuitionistic fuzzy environment. Now, the researches of decision making under intuitionistic fuzzy environment have made many meaningful results $[2,4,5,14,19,20]$.

While, in some voting, the decision makers may be inclined to some better non-candidates. Take the election of NPC for example, electors how to fill out the ballots in the election is provided by the electoral law of the People's Republic of China on the National People's Congress and Local People's Congresses as follows: "The elector may vote for or against any of the candidates that have been determined, or may instead elect any other electors or abstain from voting"[15]. Under the law, 6 people won victory for the non-candidate at the Beijing People's Congress election in 2011 [1]. If intuitionistic fuzzy set is applied into the above voting, the information of voting for non-candidates may be ignored.

Neutrosophic set proposed by Smarandache[3] is an extension of intuitionistic fuzzy set, and each element is described by the degree of membership, the degree of indeterminacy, and the degree of non-membership. 
While the neutrosophic set theory is difficult to handle the voting problems, as the sum of the three degree is greater than 1. Besides, it cannot describe the information of voting for non-candidates of the above voting. Based on this, the concept of ternary fuzzy set and ternary fuzzy number are introduced.

\section{The concept of fuzzy set and intuitionistic fuzzy set}

Zadeh [9] first introduced the fuzzy set in 1965 as follows:

Definition 1. Let $X$ be nonempty set. Then, a fuzzy set is defined by

$$
F=\left\{\left\langle x, \mu_{F}(x)\right\rangle \mid x \in X\right\},
$$

where $\mu_{F}: X \rightarrow[0,1]$ is a membership function of fuzzy set, and $\mu_{F}(x)$ is the membership degree of $x \in X$.

Atanassov [7, 8] extended the fuzzy set and proposed the concept of intuitionistic fuzzy set as follows:

Definition 2. Let $X$ be a nonempty set. Then, a intuitionistic fuzzy set is defined by

$$
\tilde{A}=\left\{\left\langle x, \mu_{\tilde{A}}(x), v_{\tilde{A}}(x)\right\rangle \mid x \in X\right\},
$$

where the functions $\mu_{\tilde{A}}: \rightarrow[0,1]$ and $\nu_{\tilde{A}}: \rightarrow[0,1]$ are the membership degree and nonmembership degree of the element $x \in X$, and $\mu_{\tilde{A}}$ and $v_{\tilde{A}}$ satisfy the following condition:

$$
0 \leq \mu_{\tilde{A}}(x)+v_{\tilde{A}}(x) \leq 1 .
$$

In addition, $\pi_{\tilde{A}}(x)=1-\mu_{\tilde{A}}(x)-v_{\tilde{A}}(x)$ denotes the indeterminacy degree of the element $x \in X$.

Remark 1. Because the intuitionistic fuzzy set is composed by the membership degree and nonmembership degree, so the intuitionistic fuzzy set can be called a binary fuzzy set. While the nonmembership degree $v_{\tilde{A}}=0$, the intuitionistic fuzzy set degenerates into a fuzzy set.

Smarandache [3] proposed neutrosophic set, which is an extension of intuitionistic fuzzy set.

Definition 3. Let $U$ be an universe of discourse. Then the neutrosophic set $A$ is an object having the form

$$
A=\left\{\left\langle x, \mu_{A}(x), v_{A}(x), \omega_{A}(x)\right\rangle \mid x \in X\right\},
$$

where the functions

$$
\left.\mu_{A}(x), v_{A}(x), \omega_{A}(x): U \rightarrow\right]^{-} 0,1^{+}[
$$

define respectively the degree of membership, the degree of indeterminacy, and the degree of nonmembership of the element $x \in X$ to the set $A$ with the condition:

$$
{ }^{-} 0 \leq \sup _{A}(x)+\operatorname{supv}_{A}(x)+\sup _{A}(x) \leq 3^{+} .
$$

\section{The mathematical model of ternary fuzzy set}

\subsection{Ternary fuzzy set}

The membership degree, nonmembership degree, indeterminacy degree of both intuitionistic fuzzy set and neutrosophic set can describe the support information, opposition information and abstention information of the candidates in many voting. In order to describe the information of voting for non-alternatives, the concept of ternary fuzzy set is given, which is an extension of intuitionistic fuzzy set.

Definition 4. Let $X$ be a nonempty set. Then, a ternary fuzzy set is defined by

$$
\tilde{\tilde{A}}=\left\{\left\langle x, \mu \tilde{\tilde{A}}(x), v_{\tilde{A}}(x), \pi \tilde{\tilde{A}}(x)\right\rangle \mid x \in X\right\},
$$

where

$$
\begin{gathered}
\mu_{\tilde{\tilde{A}}}: X \rightarrow[0,1], x \in X \\
\nu_{\tilde{A}}: X \rightarrow[0,1], x \in X \\
\pi_{\tilde{A}}: X \rightarrow[0,1], x \in X
\end{gathered}
$$

and they satisfy the following condition:

$$
0 \leq \mu_{\tilde{\tilde{A}}}(x)+v_{\tilde{A}}(x)+\pi \tilde{\tilde{A}}(x) \leq 1, \forall x \in X .
$$

$\mu_{\tilde{\tilde{A}}}, \nu_{\tilde{A}}$ define the membership degree and nonmembership degree of $\tilde{\tilde{A}} \cdot \rho_{\tilde{A}}=\mu_{\tilde{A}}+v_{\tilde{A}}$ denotes the candidate degree, $\pi \tilde{\tilde{A}}$ defines the non-candidate degree. Then,

$$
\left\{\left\langle x, \rho_{\tilde{A}}(x), \pi \tilde{\tilde{A}}(x)\right\rangle \mid x \in X\right\}
$$

defines the intuitionistic fuzzy set of candidate. In addition, $\tau_{\tilde{\tilde{A}}}=1-\mu_{\tilde{\tilde{A}}}-v_{\tilde{\tilde{A}}}-\pi_{\tilde{A}}$ defines the indeterminacy degree of $\tilde{\tilde{A}}$.

Remark 2. While $\pi_{\tilde{A}}=0$, the ternary fuzzy set $\tilde{\tilde{A}}$ becomes a intuitionistic fuzzy set

$$
\left\{\left\langle x, \mu \tilde{\tilde{A}}(x), \nu_{\tilde{A}}(x)\right\rangle \mid x \in X\right\} ;
$$

while $\nu_{\tilde{\tilde{A}}}=\pi \tilde{\tilde{A}}=0$, the ternary fuzzy set $\tilde{\tilde{A}}$ becomes a fuzzy set $\{\langle x, \mu \tilde{\tilde{A}}(x)\rangle \mid x \in X\}$. Hence, Both fuzzy set 


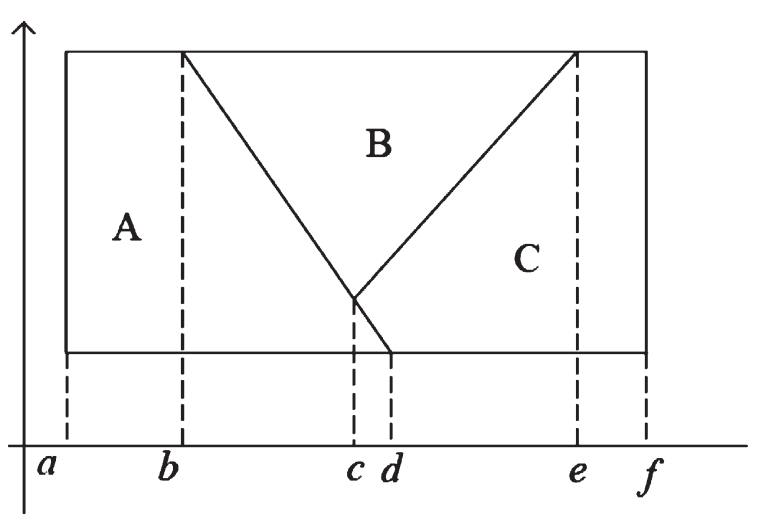

Fig. 1. A sample of ternary fuzzy set.

and intuitionistic fuzzy set are the special case of the ternary fuzzy set. But the operations of fuzzy set are different from intuitionistic fuzzy set and ternary fuzzy set.

Example 1. As shown in Fig. 1, suppose $A, B, C$ are three nonempty sets in the 2-dimensional vector space, and let $X=A \cup B \bigcup C$. For any given element $x=$ $\left(\mathrm{x}_{1}, \mathrm{x}_{2}\right) \in X$, the value of $\mathrm{x}_{1}$ is known, and the value of $\mathrm{x}_{2}$ is unknown. For a unknown set $F$ satisfies

$$
A \subset F \subset A \bigcup B, A \neq F \neq A \bigcup B .
$$

Then a ternary fuzzy set is given by

$$
\tilde{\tilde{F}}=\left\{\left\langle x, \mu_{\tilde{F}}(x), v_{\tilde{F}}(x), \pi_{\tilde{F}}(x)\right\rangle \mid x \in X\right\},
$$

where

$$
\begin{aligned}
& \mu_{\tilde{F}}(x)=\left\{\begin{array}{cc}
1 & a \leq L(x) \leq b \\
0.7 & b<L(x) \leq c \\
0.2 & c<L(x) \leq d \\
0.1 & d<L(x) \leq e \\
0 & e<L(x) \leq f,
\end{array}\right. \\
& \nu_{\tilde{F}}(x)=\left\{\begin{array}{c}
0 \quad a \leq L(x) \leq b \\
0.2 b<L(x) \leq c \\
0.4 c<L(x) \leq d \\
0.3 d<L(x) \leq e \\
0 \quad e<L(x) \leq f,
\end{array}\right. \\
& \pi_{\tilde{F}}(x)=\left\{\begin{array}{cc}
0 & a \leq L(x) \leq c \\
0.1 & c<L(x) \leq d \\
0.5 & d<L(x) \leq e \\
1 & e<L(x) \leq f .
\end{array}\right.
\end{aligned}
$$

For any given element $x \in X$, we have

$$
0 \leq \mu_{\tilde{F}}(x)+v_{\tilde{F}}(x)+\pi_{\tilde{F}}(x) \leq 1,
$$

and,

$$
\rho_{\tilde{F}}=\mu_{\tilde{F}}+v_{\tilde{F}}=\left\{\begin{array}{cc}
1 & a \leq L(x) \leq b \\
0.9 & b<L(x) \leq c \\
0.6 & c<L(x) \leq d \\
0.4 & d<L(x) \leq e \\
0 & e<L(x) \leq f .
\end{array}\right.
$$

Then, $\quad\left\{\left\langle x, \rho_{\tilde{F}}(x), \pi \tilde{\tilde{F}}(x)\right\rangle \mid x \in X\right\}$ is a intuitionistic fuzzy candidate set with respect to $\tilde{\tilde{F}}$, and the indeterminacy degree of element $x \in X$ is given by

$$
\tau_{\tilde{F}}(x)=\left\{\begin{array}{cc}
0 & a \leq L(x) \leq b \\
0.1 & b<L(x) \leq c \\
0.3 & c<L(x) \leq d \\
0.1 & d<L(x) \leq e \\
0 & e<L(x) \leq f .
\end{array}\right.
$$

Next, the operation and property of ternary fuzzy sets are discussed.

Definition 5. Let $X$ be a nonempty set. The three ternary fuzzy sets defined on $X$ are given by

$$
\begin{gathered}
\tilde{\tilde{A}}=\left\{\left\langle x, \mu_{\tilde{A}}(x), v_{\tilde{A}}(x), \pi \tilde{\tilde{A}}(x)\right\rangle \mid x \in X\right\} \\
\tilde{\tilde{A}}_{1}=\left\{\left\langle x, \mu_{\tilde{\tilde{A}}_{1}}(x), v_{\tilde{\tilde{A}}_{1}}(x), \pi \tilde{\tilde{A}}_{1}(x)\right\rangle \mid x \in X\right\} \\
\tilde{\tilde{A}}_{2}=\left\{\left\langle x, \mu_{\tilde{\tilde{A}}_{2}}(x), v_{\tilde{\tilde{A}}_{2}}(x), \pi_{\tilde{\tilde{A}}_{2}}(x)\right\rangle \mid x \in X\right\}
\end{gathered}
$$

Then the operations of the ternary fuzzy sets are defined by

(1) $\tilde{\tilde{A}}^{c}=\left\{\left\langle x, \nu_{\tilde{A}}(x), \mu \tilde{\tilde{A}}(x), \pi \tilde{\tilde{A}}(x)\right\rangle\right\}$;

(2) $\tilde{\tilde{A}}_{1} \bigcup \tilde{\tilde{A}}_{2}=\left\{\left\langle x, \max \left\{\mu_{\tilde{A}_{1}}(x), \mu_{\tilde{\tilde{A}}_{2}}(x)\right\}\right.\right.$, $\left.\left.\min \left\{v_{\tilde{\tilde{A}}_{1}}(x), v_{\tilde{A}_{2}}(x)\right\}, \min \left\{\pi \tilde{\tilde{A}}_{1}(x), \pi_{\tilde{\tilde{A}}_{2}}(x)\right\}\right\rangle\right\} ;$

(3) $\tilde{\tilde{A}}_{1} \cap \tilde{\tilde{A}}_{2}=\left\{\left\langle x, \min \left\{\mu_{\tilde{A}_{1}}(x), \mu_{\tilde{A}_{2}}(x)\right\}\right.\right.$, $\left.\left.\max \left\{v_{\tilde{\tilde{A}}_{1}}(x), v_{\tilde{A}_{2}}(x)\right\}, \min \left\{\pi \tilde{\tilde{A}}_{1}(x), \pi_{\tilde{A}_{2}}(x)\right\}\right\rangle\right\} ;$

(4) $\tilde{\tilde{A}}_{1}+\tilde{\tilde{A}}_{2}=\left\{\left\langle x, 1-\left(1-\mu \tilde{\tilde{A}}_{1}(x)\right)(1-\right.\right.$ $\left.\left.\left.\mu \tilde{\tilde{A}}_{2}(x)\right), v_{\tilde{A}_{1}}(x) v_{\tilde{A}_{2}}(x), \pi \tilde{\tilde{A}}_{1}(x) \pi \tilde{\tilde{A}}_{2}(x)\right\rangle\right\} ;$

(5) $\tilde{\tilde{A}}_{1} \cdot \tilde{\tilde{A}}_{2}=\left\{\left\langle x, \mu_{\tilde{\tilde{A}}_{1}}(x) \mu_{\tilde{\tilde{A}}_{2}}(x), 1-(1-\right.\right.$ $\left.\left.\left.\nu_{\tilde{A}_{1}}(x)\right)\left(1-v_{\tilde{A}_{2}}(x)\right), \pi \tilde{\tilde{A}}_{1}(x) \pi \tilde{\tilde{A}}_{2}(x)\right\rangle\right\}$.

Property 1. The above operations of ternary fuzzy set are closed. 


\section{Proof.}

(1) It is obvious that $\tilde{\tilde{A}}^{c}$ is also a ternary fuzzy set.

(2) Let $\tilde{\tilde{A}}_{1}, \tilde{\tilde{A}}_{2}$ be two ternary fuzzy sets. For any given $x \in X$, suppose

$$
\max \left\{\mu_{\tilde{A}_{1}}, \mu_{\tilde{A}_{2}}\right\}=\mu_{\tilde{A}_{1}} \cdot
$$

Because

$$
\min \left\{v_{\tilde{A}_{1}}, v_{\tilde{A}_{2}}\right\} \leq v_{\tilde{A}_{1}}, \min \left\{\pi \tilde{\tilde{A}}_{1}, \pi_{\tilde{A}_{2}}\right\} \leq \pi \tilde{\tilde{A}}_{1},
$$

so we have

$$
\begin{gathered}
\max \left\{\mu_{\tilde{A}_{1}}, \mu_{\tilde{A}_{2}}\right\}+\min \left\{v_{\tilde{A}_{1}}, v_{\tilde{A}_{2}}\right\}+\min \left\{\pi \tilde{\tilde{A}}_{1}, \pi_{\tilde{A}_{2}}\right\} \\
\leq \mu_{\tilde{A}_{1}}+v_{\tilde{A}_{1}}+\pi \tilde{\tilde{A}}_{1} \leq 1
\end{gathered}
$$

i.e. $\tilde{\tilde{A}}_{1} \bigcup \tilde{\tilde{A}}_{2}$ is a ternary fuzzy set. Similarly, $\tilde{\tilde{A}}_{1} \cap \tilde{\tilde{A}}_{2}$ is also a ternary fuzzy set.

(3) Let $\tilde{\tilde{A}}_{1}, \tilde{\tilde{A}}_{2}$ be two ternary fuzzy sets. As

$$
1-\mu \tilde{\tilde{A}}_{i} \geq v_{\tilde{A}_{i}}+\pi \tilde{\tilde{A}}_{i}, i=1,2,
$$

we have

$0 \leq 1-\left(1-\mu_{\tilde{A}_{1}}\right)\left(1-\mu \tilde{\tilde{A}}_{2}\right)+v \tilde{\tilde{A}}_{1} v_{\tilde{A}_{2}}+\pi \tilde{\tilde{A}}_{1} \pi_{\tilde{A}_{2}} \leq$ $1-\left(v \tilde{\tilde{A}}_{1}+\pi \tilde{\tilde{A}}_{1}\right)\left(v \tilde{\tilde{A}}_{2}+\pi \tilde{\tilde{A}}_{2}\right)+v_{\tilde{A}_{1}} v_{\tilde{A}_{2}}+\pi \tilde{\tilde{A}}_{1} \pi \tilde{\tilde{A}}_{2}=$ $1-v_{\tilde{A}_{1}} \pi \tilde{\tilde{A}}_{2}-v_{\tilde{A}_{2}} \pi \tilde{\tilde{A}}_{1} \leq 1$

i.e. $\tilde{\tilde{A}}_{1}+\tilde{\tilde{A}}_{2}$ is a ternary fuzzy set.

Property 2 . Let $\tilde{\tilde{A}}, \tilde{\tilde{A}_{1}}, \tilde{\tilde{A}}_{2}$ be three ternary fuzzy sets respectively. Then, we have

(1) $\left(\tilde{\tilde{A}}^{c}\right)^{c}=\tilde{\tilde{A}}$;

(2) $\left(\tilde{\tilde{A}}_{1} \bigcup \tilde{\tilde{A}}_{2}\right)^{c}=\tilde{\tilde{A}}_{1}{ }^{c} \cap \tilde{\tilde{A}}_{2}{ }^{c}$, $\left(\tilde{\tilde{A}}_{1} \cap \tilde{\tilde{A}}_{2}\right)^{c}=\tilde{\tilde{A}}_{1}{ }^{c} \cup \tilde{\tilde{A}}_{2}{ }^{c} ;$

(3) $\left(\tilde{\tilde{A}}_{1}+\tilde{\tilde{A}}_{2}\right)^{c}=\tilde{\tilde{A}}_{1}{ }^{c} \cdot \tilde{\tilde{A}}_{2}{ }^{c}$, $\left(\tilde{\tilde{A}}_{1} \cdot \tilde{\tilde{A}}_{2}\right)^{c}=\tilde{\tilde{A}}_{1}{ }^{c}+\tilde{\tilde{A}}_{2}{ }^{c}$.

Proof. It is easy to get above conclusions by the use of the operations.

\subsection{Ternary fuzzy number}

Based on ternary fuzzy set, a ternary fuzzy number is given by

$$
\alpha=\left\langle\mu_{\alpha}, v_{\alpha}, \pi_{\alpha}\right\rangle
$$

where

$$
\mu_{\alpha}, v_{\alpha}, \pi_{\alpha} \in[0,1], \mu_{\alpha}+v_{\alpha}+\pi_{\alpha} \in[0,1] .
$$

In some voting, $\mu_{\alpha}, v_{\alpha}$ can denote support information, opposition information of the voting. $\pi_{\alpha}$ can denote the information of voting for non-candidates. Denote $\rho_{\alpha}=\mu_{\alpha}+v_{\alpha},\left(\rho_{\alpha}, \pi_{\alpha}\right)$ is a intuitionistic fuzzy candidate number with respect to $\alpha$. Then, $\tau_{\alpha}=1-$ $\left(\rho_{\alpha}+\pi_{\alpha}\right)$ can denote the abstention information.

Take the voting of NPC deputies for example, the electors may vote for or against any of the candidates that have been determined, or may instead elect any other electors or abstain from voting. So that, ternary fuzzy number is given to describe above voting information as follows: For example, 10 people participated in the election, and $\alpha$ is the only candidate. The voting result is that 4 people vote for $\alpha, 2$ people against $\alpha, 1$ people abstain from voting and 1 people vote for other non-candidate. The electoral result can be denoted by

$$
\alpha=\langle 0.4,0.2,0.3\rangle \text {. }
$$

Remark 3. Though the neutrosophic set was given by the degree of membership, the degree of indeterminacy, and the degree of non-membership, it is difficult to handle the voting problems, as the sum of the three degree is greater than 1 .

For any given ternary fuzzy number $\alpha=\left\langle\mu_{\alpha}, v_{\alpha}, \pi_{\alpha}\right\rangle$, it is can be measured simply by a score function as follows:

$$
s(\alpha)=\mu_{\alpha}-v_{\alpha} .
$$

If $\mu_{\alpha}=1, v_{\alpha}=0$, i.e. $\alpha=\langle 1,0,0\rangle, s(\alpha)$ reaches a maximum value of 1 ; If $\mu_{\alpha}=0, v_{\alpha}=1$, i.e. $\alpha=$ $\langle 0,1,0\rangle, s(\alpha)$ reaches a minimum value of -1 .

Let $\alpha, \beta$ be two ternary fuzzy numbers. Then the relation $\alpha \prec \beta$ is valid iff $s(\alpha)<s(\beta)$.

For example, if $\quad \alpha=\langle 0.5,0.3,0.2\rangle, \beta=$ $\langle 0.5,0.2,0.1\rangle$. Then

$$
0.2=s(\alpha)<s(\beta)=0.3 \Rightarrow \alpha \prec \beta .
$$

However, if $\quad s(\alpha)=s(\beta), \quad$ such as $\quad \alpha=$ $\langle 0.5,0.2,0.2\rangle, \beta=\langle 0.5,0.2,0.1\rangle$, it is difficult to get the relation " $\prec$ " between $\alpha$ and $\beta$.

Let $\alpha=\left\langle\mu_{\alpha}, v_{\alpha}, \pi_{\alpha}\right\rangle$ and $\beta=\left\langle\mu_{\beta}, v_{\beta}, \pi_{\beta}\right\rangle$ be two ternary fuzzy numbers. Based on the above score function, a comprehensive measure method of ternary fuzzy numbers is given:

(1) If $s(\alpha)<s(\beta)$, we have $\alpha \prec \beta$.

(2) If $s(\alpha)=s(\beta)$,

(a) While $\rho_{\alpha}<\rho_{\beta}$, we have $\alpha \prec \beta$.

(b) While $\rho_{\alpha}=\rho_{\beta}$ i.e. $\mu_{\alpha}=\mu_{\beta}, v_{\alpha}=v_{\beta}$, we have

(i) $\pi_{\alpha}>\pi_{\beta} \Rightarrow \alpha \prec \beta$;

(ii) $\pi_{\alpha}=\pi_{\beta} \Rightarrow \alpha=\beta$. 
Let $\alpha=\langle 0.5,0.2,0.2\rangle, \beta=\langle 0.5,0.2,0.1\rangle$. So we have $\pi_{\alpha}>\pi_{\beta} \Rightarrow \alpha \prec \beta$ by the use of the above method.

\section{An example for voting}

Take a voting of a region's deputies for example. There are 4 candidates $(A, B, C, D)$ determined, and 3 deputies should be chosen. The ballot is shown in Table 1, and provisions of the voting are given as follows:

(1) Vote for no more than 3;

(2) Draw " $\bigcirc$ "in front of the name voting for, or Draw " $x$ "in front of the name against;

(3) If you want replace the candidate with a noncandidate, write in the non-candidate's name behind the candidate's name to be replaced and draw " $\bigcirc$ " in front of the candidate's name replaced;

(4) Do not mark with any symbol if abstain from voting.

In the end, there are 100 valid ballots. The final vote count of candidate is given as follows:

A: 70 people vote for, 20 people against, 4 people abstain from and 6 people vote for non-candidates (such that 4 people vote for $E$ and 2 people vote for $F$ ).

$B$ : 30 people vote for, 28 people against, 2 people abstain from and 40 people vote for non-candidates (such that 30 people vote for and 10 people vote for $F)$.

$C$ : 55 people vote for, 25 people against, 5 people abstain from and 15 people vote for non-candidates (such that 15 people vote for $E$ ).

$D$ : 55 people vote for, 25 people against, 0 people abstain from and 20 people vote for non-candidates (such that 19 people vote for $E$ and 1 people vote for $F)$.

The final vote count of the four candidates can be denoted by the ternary fuzzy numbers:

Table 1

The ballot

\begin{tabular}{ccc}
\hline Symbol & Candidates'name & Non-candidates'name \\
\hline $\mathrm{A}$ & \\
$\mathrm{B}$ & \\
$\mathrm{C}$ & \\
$\mathrm{D}$ & \\
\hline
\end{tabular}

$$
\begin{gathered}
A=\langle 0.7,0.2,0.06\rangle, \pi_{A}=0.06=\frac{0.04}{E}+\frac{0.02}{F} ; \\
B=\langle 0.3,0.28,0.4\rangle, \quad \pi_{B}=0.4=\frac{0.3}{E}+\frac{0.1}{F} ; \\
C=\langle 0.55,0.25,0.15\rangle, \quad \pi_{C}=0.15=\frac{0.15}{E}+\frac{0}{F} ; \\
D=\langle 0.55,0.25,0.2\rangle, \quad \pi_{D}=0.2=\frac{0.19}{E}+\frac{0.01}{F} .
\end{gathered}
$$

At the same time, we can not ignore the two non-candidates $(E, F)$. So the final vote count of $E$ and $F$ can be denoted by the ternary fuzzy numbers:

$$
E=\langle 0.68,0,0\rangle, F=\langle 0.13,0,0\rangle .
$$

Accordance with electoral provisions, the top three candidates and non-candidates with higher percentage of votes obtained win the qualifications. It is obvious that

$$
\mu_{A}>\mu_{E}>\mu_{C}=\mu_{D}>\mu_{B}>\mu_{F} .
$$

So the candidates $A$ and non-candidates $B$ win two of the three qualifications. While the candidates $C$ and $D$ have the same percentage of votes, the traditional approach is to re-vote for the two candidates, which is a time-consuming work. Now, we can measure the ternary fuzzy numbers $C$ and $D$ to determine the last qualification of deputy:

$$
\mu_{C}=\mu_{D}, v_{C}=v_{D}, \pi_{D}>\pi_{C} \Rightarrow D \prec C .
$$

Then, $C$ win the last one qualification of deputy. So, the proposed approach of ternary fuzzy numbers improves the efficiency of elections.

\section{Conclusions}

In some voting, both fuzzy set and intuitionistic fuzzy set are difficult to deal with the information of voting for non-candidates. Consequently, the concept of ternary fuzzy set and ternary fuzzy number are proposed in this paper, which extend the fuzzy set and intuitionistic fuzzy set. Furthermore, the information aggregation approach of ternary fuzzy set will be given, and decision making models of ternary fuzzy sets will be further studied.

\section{Acknowledgments}

This work was supported by the National Natural Science Foundation of China (No. 61073121), the Natural 
Science Foundation of Hebei Province of China (Nos. F2015402033, A2012201033).

\section{References}

[1] Beijing districts and counties of the township people's congress deputies election: 6 people won the election as non-candidates, http://news.xinhuanet.com/politics/2011-11/16/c122287108. htm, 2011.

[2] D.F. Li, Mathematical-programming approach to matrix games with payoffs represented by Atanassov's interval-valued intuitionistic fuzzy sets, IEEE Transactions on Fuzzy Systems 18(6) (2010), 1112-1128.

[3] F. Smarandache, A Unifying Field in Logics: Neutrsophic Logic. Neutrosophy, Neutrosophic Set, Neutrosophic Probability(fourth edition), American Research Press, 2005.

[4] G. Beliakov, H. Bustince, S. James, T. Calvo and J. Fernandez, Aggregation for Atanassov's intuitionistic and interval valued fuzzy sets: The median operator, IEEE Transactions on Fuzzy Systems 20(3) (2012), 487-498.

[5] H.L. Jiang, J.M. He, Y.M. Zhuang and Y.F. Zhang, Approach to group decision making based on intuitionistic fuzzy sets and evidence theory, Control and Decision 27(5) (2012), 752-756.

[6] J. Ma, G.Q. Zhang and J. Lu, A method for multiple periodic factor prediction problems using complex fuzzy sets, IEEE Transactions on Fuzzy Systems 20(1) (2012), 32-45.

[7] K. Atanassov, Intuitionistic fuzzy sets, VII ITKR's Session, 1983.

[8] K. Atanassov, Intuitionistic fuzzy sets, Fuzzy Sets and Systems 20 (1986), 87-96.
[9] L.A. Zadeh, Fuzzy sets, Information and Control 8 (1965), 338-353.

[10] M. Galar, J. Fernandez, G. Beliakov and H. Bustince, Interval-valued fuzzy sets applied to stereo matching of color images, IEEE Transactions on Image Processing 20(7) (2011), 1949-1961.

[11] S.J. Chuu, Group decision-making model using fuzzy multiple attributes analysis for the evaluation of advanced manufacturing technology, Fuzzy Sets and Systems 160(5) (2009), 586-602.

[12] S.K. De, R. Biswas and A.R. Roy, Some operations on intuitionistic fuzzy sets, Fuzzy Sets and Systems 114 (2000), 477-484.

[13] S.M. Taheri and R. Azizi, On information energy of the image fuzzy sets, Information Sciences 177(18) (2007), 3871-3881.

[14] T. Chaira, Intuitionistic fuzzy segmentation of medical images, IEEE Transactions on Biomedical Engineering 57(6) (2010), 1430-1436.

[15] The Electoral Law of the People's Republic of China on the National People's Congress and Local People's Congresses, China Legal Publishing House, 2010.

[16] Y.R. Syau and L.E. Stanley, Preincavity and fuzzy decision making, Fuzzy Sets and Systems 155(3) (2005), 408-424.

[17] Z.S. Xu, Intuitionistic fuzzy aggregation operators, IEEE Transactions on Fuzzy Systems 15 (2007), 1179-1187.

[18] Z.S. Xu, Multi-person multi-attribute decision making models under intuitionistic fuzzt environment, Fuzzy Optimization and Decision Making 6 (2007), 221-236.

[19] Z.S. Xu, Intuitionistic Fuzzy Information Aggregation Theory and Applications, Science Press, 2008.

[20] Z.S. Xu, Intuitionistic fuzzy multi attribute decision making: An interactive method, IEEE Transactions on Fuzzy Systems 20(3) (2012), 514-525. 